\title{
CHEMICAL AND BIOLOGICAL EVOLUTION IN SPACE
}

\author{
J. Mayo Greenberg, Peter Weber and Willem Schutte \\ Laboratory Astrophysics, University of Leiden, P.O. Box 9504, \\ 2300 RA Leiden, The Netherlands
}

\section{ABSTRACT}

Astronomical Infrared spectra are used to confirm the existence of complex organic molecules produced by ultraviolet photoprocessing of interstellar grain mantles. This material is shown to be the major component of the interstellar grains between the sun and the galactic center and, by inference, constitutes more than 10 million solar masses - or close to one part in a thousand of the entire mass of the milky way galaxy. It may be demonstrated that the primitive chemistry of the earth's surface was dominated by these extraterrestrial molecules after aggregated into comets if the rate of comet impacts with the earth was comparable with that required to account for the extinction of species over the past 300 million years.

Ultraviolet irradiation of bacterial spores has been studied for the first time under simulated interstellar conditions. The inactivation time predicted for the less dense regions of space is at most several hundred years. Within molecular clouds it is shown on theoretical and experimental grounds that this time may be extended to tens of million of years which is the estimated time required for thelr transport from one solar system to another by a molecular cloud. However survival of spores during their intial exposure to the solar ultraviolet presents a problem for panspermia because it requires that in the process of ejection from the earth's surface they must be enclosed within a cocoon (or mantle) of ultraviolet absorbing materlal of $\sim 0.6 \mu \mathrm{m}$ thickness. Thus, although panspermia can not be rejected on the basis of lack of interstellar survival there may remain insurmountable obstacles to its occurring because of the very spectal protective shield requirements during ejection from its planetary source.

\section{INTRODUCTION}

The interstellar medium provides the conditions for a wide range of chemical and physical processes leading both to the formation and destruction of molecules out of the constituent atoms. The cosmically abundant condensable elements: $0, \mathrm{C}, \mathrm{N}, \mathrm{Mg}, \mathrm{Si}, \mathrm{Fe}, \mathrm{S}$, in combination with each other and with the most abundant element hydrogen, exist both in gaseous and solid form. Since the $1960^{\prime} \mathrm{s}$ a larger and larger number of molecules have been identified in the gas phase, primarily by radio astronomical techniques. Identification of molecular constituents in the solid phase has proceeded much more slowly although progress has accelerated recently wh improvements in infrared techniques used to study astronomical absorption and enission spectra. Several excellent reviews of the status of molecules in the gas show the extent of our current knowledge of the presence of some 60 molecules in various 1sotopic configurations in both neutral and ionized states $[1,2]$.

We shall focus our attention here on the solid particles in space, otherwise known as interstellar grains or dust. The evolution of these particles which results from interactions with the gaseous atans and molecules, with ultraviolet radiation, with each other and perhaps also with cosmic rays leads to the production of extremely complex organic molecules within the dust. Identification has been made of some smaller molecules in the grains but the preclse identification of the complex molecules by purely astronomical methods will perhaps never be possible. However, current infrared observations combined with laboratory and theoretical studies give credence to their presence - Indeed in enormous quantities - in space. How these molecules are formed and their quantities estimated will be outlined in this paper.

There is both a similarity and an essential difference between interstellar grain evolution and interstellar effects on living organisms. In the former, the effects of ultraviolet radiation lead to the formation of complex organic molecules from simple molecules; while in the latter the ultraviolet radiation leads to the destruction and rearrangement of already existing complex molecules. In elther case the process is initiated 
by the breaking of a molecular bond or the ionization or excitation by an ultraviolet photon.

The interstellar mediun.

The basic ingredients of the interstellar medium are the atoms, molecules, ions, ultraviolet radiation and cosmic rays. We scale the chemically active atoms into three groups according to their abundances: 1) hydrogen; 2) oxygen, carbon, nitrogen; 3) magnesium, silicon, iron, sulfur. Hydrogen is by far the most abundant and we shall scale everything relative to it. on this basis, group (2), which we call the organics are in total down by a factor of $\sim 10^{-3}$ with $0: C: N=6.8: 3.7: 1.2\left(10^{-4}\right)$ and group (3) are in total down by a factor of $\sim 10^{-4}$ with Mg:Si:Fe:S $=3.4: 3.2: 2.6: 2.8\left(10^{-5}\right)$ [3]. We could, of course, include $S$ anong the organics although its abundance is significantly lower.

The matter in space is very unevenly distributed, with many orders of magnitude variety in density. The hydrogen density in clouds can be observed to be as high as $n_{\mathrm{H}}=10^{6}-10^{\prime} \mathrm{cm}^{-3}$ and, in the intercloud medium, as low as $\mathrm{n}_{\mathrm{H}} \simeq 10^{-1} \mathrm{~cm}^{-3}$. Clouds $\mathrm{Qf}^{\mathrm{f}}$ density $\mathrm{n}_{\mathrm{H}} \approx 10-20 \mathrm{~cm}^{-3}$ are called diffuse and, since clouds of density $\mathrm{n}_{\mathrm{H}} 210^{2} \mathrm{~cm}^{-3}$ contain hydrogen in molecular form, they are called molecular clouds. It is in such clouds that one also detects $C O$ in substantial abundance. The overall density of hydrogen is $\mathrm{n}_{\mathrm{H}}=1 \mathrm{~cm}^{-3}[4]$.

The temperature of the gas in neutral regions is in the range $10-100 \mathrm{~K}$. The bulk of the interstellar dust is in particles of characteristic semi-thickness (which we call a radius a for convenience) $a \simeq 0.1-0.15 \mu \mathrm{m}$. These particles consist of small silicate cores $\left(a_{c} \simeq 0.05 \mu \mathrm{m}\right)$ with mantles made up of the organics and are henceforth called coremantle particles ( $c-m$ ). The number density of these core-mantle particles relative to hydrogen is $\mathrm{n}_{\mathrm{c}-\mathrm{m}}=10^{-12} \mathrm{n}_{\mathrm{H}}$. In additiun to these core-mantle particles there are much larger numbers of much smaller particles $(a<0.01 \mu \mathrm{m})$ of carbon, sllicates and perhaps extremely small particles $(a<0.02 \mu \mathrm{m})$ of unknown chemical composition [5, 6, 7]. These will not be considered here in detall. Since the interstellar grains are very efficient in attenuating light they are the cause for the appearance of the dark patches in the sky which delineate the denser clouds optically.

Most of the ultraviolet radiation in space comes from distant stars. Within clouds this ultraviolet radiation is attenuated by the dust. However, within clouds stars are born and the winds from young stars are an indirect source of ultraviolet. Cosmic ray protons also give rise to ultraviolet (Ly $\alpha$ ) photons whose flux is significant within clouds [8]. In diffuse regions outside of the clouds the flux of photons with $\lambda<2000 \AA 1$ s $\Phi \simeq 10^{8} \mathrm{hv} \mathrm{cm} \mathrm{s}^{-1}$ [5]. Within clouds the sum of the ultraviolet radiation from without and within may be reduced from this amount but probably never less than by a factor between $10^{-3}$ and $10^{-4}$, with the latter being a reasonable lower $11 \mathrm{mit}$. In the interstellar medium the energy density between $2000 \AA$ and $3000 \AA$ is about equal to that between $1000 \AA$ and $2000 \AA[9]$.

Chemical Evolution of Grains and Gas

The interstellar medium is highly dynamic. Not only are the clouds moving about rapidly they are continually evolving. The less dense regions, whether by coagulation or as a result of compression by shocks induced by star formation, become denser. Within clouds of sufficient density, star formation may take place with, in many cases - particularly where large stars are born - a violent ejection of the remaining matter back to the surrounding diffuse regions. The interstellar medium (ISM) loses mass each time a star is born, but loss of mass from stars during their evolution is a source of new material [10], The rate at which th1s occurs, in other words, the turnover time for the ISM is about $5 \times 10^{9}$ years. Since, all the material which goes to make up a star is reduced to its atomic constituents, this is the maximum Iifetime of any part of the ISM including the dust grains [11].

In dense clouds molecules are produced in the gas primarily via fon-molecule reactions. At the same time, atons and molecules collide with grains and interact to form mantles of new molecules on the grain. Accompanying the accretion, ultraviolet photons penetrate the grains producing free radicals and molecules. The free radicals are frozen in at the previling temperature of $10 \mathrm{~K}$ thus storing chemical energy in the grains. When grains are impulsively elevated in temperature by $\Delta T \cong 15 \mathrm{~K}$ an explosive reaction occurs among these radicals and the grain mantle evaporates, replenishing the gas. Within most molecular clouds the process of accretion and explosion leads to a quasi-steady state (over a large part of the cloud lifetime) between gas and grains even though the entire process is time dependent [12]. However, there is a gradual build-up of very complex organic material on the grains which is called organfc refractory (nonvolatile as compared with simple ices) or yellow stuff residue - becuase of 1ts color as seen in laboratory created samples resulting from ultraviolet photoprocessing and warmup of ice mixtures at $10 \mathrm{~K}[13]$. The residue appears yellow because it absorbs strongly in the blue and ultraviolet.

When grains are blown back into the diffuse medium, first evaporation and subsequent erosion - primarily by supernova explostons - removes the volatile mantles and reduces the organic refractory mantle thickness [14]. However, when the grains are returned, along with 
their supporting clouds, to the molecular clond phase they replenish the lost materials by newly accreted molecules. This cycle repeats itself te least once every $2 \times 10^{8}$ years so that during the $5 \times 10^{9}$ year iffetime it occurs 25 times or more. Therefore the grains observed in diffuse clouds are also in a quasi-steady state in which the mantles consist only of a nonvolatile residue of complex organic molecules. Since these diffuse cloud grains provide the nuclei for accretion within molecular clouds, all core-mantle grains are presumed to consist, minimally, of a silicate core plus organic refractory mantle and in molecular clouds there is an additional mantle of volatile ices. In the next section we shall present evidence for grains of both types.

\section{Grains in diffuse clouds}

The general properties or continuun optical propertles of grains in diffuse clouds are well known [6]. However useful spectroscoplc data - and this means primarily in the infrared - is scarce. This is a selection effect, because it is not of ten that one has a strong infrared source and a sufficlent column density of grains to provide a measurable spectral feature. One example is given by viewing towards the center of our galaxy which is about 10 kpc (30 light years away) and which also has some strong infrared sources. In Fig. 1 is presented the infrared absorption spectrum for the source IRS7 in the galactic center. For comparison a laboratory absorption spectrum of an organic residue is shown.

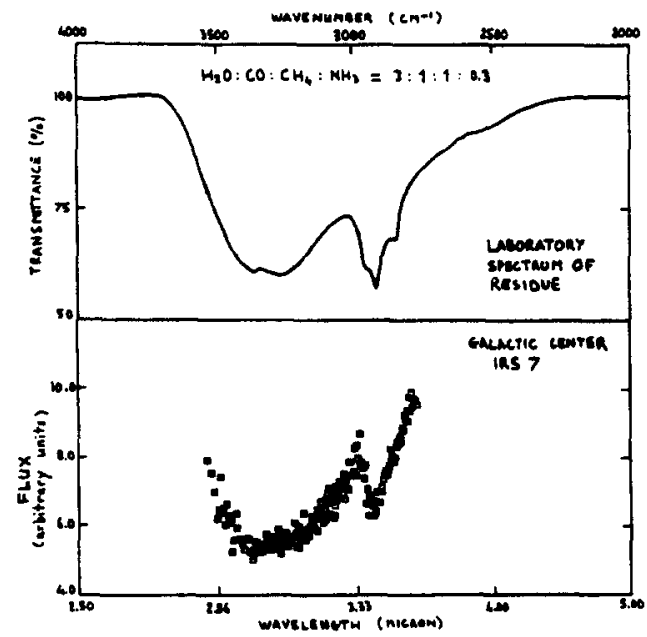

Figure 1: Infrared absorption spectrum of the source IRS 7 in the galactic center (lower curve) compared with the transmissIon spectrum (upper curve) of an organic residue resulting fror photolysis at $10-15$ $\mathrm{K}$ of a mixture of $\mathrm{H}_{2} \mathrm{O}: \mathrm{CO}: \mathrm{CH}_{4}: \mathrm{NH}_{3}=3: 1: 1: 0.3$ and wamup to room temperature.

The ratio of the strength of the $3.4 \mu \mathrm{m}$ feature to the extinction computed for a sillcate core - organic refractory grain is given by [13]

$$
\frac{\tau(3.4)}{\tau(V)}=\frac{16 \pi}{3.4} \frac{a_{2}^{3}-a_{1}^{3}}{a_{2}^{2}} \frac{\varepsilon_{2}}{\left(\varepsilon_{1}+2\right)^{2}+\varepsilon_{2}^{2}}
$$

where the radil are in microns and $a_{1}{ }_{2} a_{c}=$ radius of the silicate core, $a_{2}=a_{c-m}=$ radius of the organic residue mantle, $\varepsilon=\varepsilon,-\varepsilon_{1}$ is the complex dielectric coeffictent, which, in terms of the complex index of refraction, $m^{2}=m^{\prime}-i m "$, is defined by $\varepsilon_{1}=m^{2}-m^{\prime \prime}$, $\varepsilon_{2}=2 m^{\prime \prime} m^{\prime \prime}$.

The observed ratio for the galactic center is

$$
\left[\frac{\tau(3.4)}{\tau(V)}\right]_{\text {G.C. } .}=\frac{0.3}{30-50}=0.01-0.006
$$

where 30 is the most accepted value for the extinction to the galact1c center [15]. There are a number of reasons to regard this value as being too low and we suggest that a value closer to $50 \mathrm{might}$ be closer to the correct one [16].

For the yellow stuff spectra shown in Fig. I the value of the excess absorption at $3.4 \mu \mathrm{m}$ is $\Delta \mathrm{m}_{3.4}^{\prime \prime}=0.015$ which, with $\mathrm{a}_{1}=0.05 \mu \mathrm{m}, \mathrm{a}_{2}=0.12 \mu \mathrm{m}$ gives

$$
\left[\frac{\left.\tau(v+)^{4}\right)}{\tau(v)}\right]_{\text {Calc. }}=0.006
$$


which is within range of values observed.

We expect that the laboratory residue used here is not yet in its final state because when such residues are created in space they are further subjected to ultraviolet irradiation for billions of years in the diffuse medium. This additional photoprocessing tends to increase the $3.4 \mu \mathrm{m}$ absorption strength by "carbonization" of the organic materials. We note here that the in plied mass of yellow stuff in the galaxy is quite large. The mean density of yellow stuff along the direction to the galactic center is [5]

$$
\rho_{O . R .} \simeq \frac{4}{3} \frac{{ }^{a} c-m}{1.5 \mathrm{D}(V)} \bar{s} \simeq 1 \times 10^{-26} \mathrm{gm} \mathrm{cm}^{-3}
$$

With a mean hydrogen density of $\sim 2.5 \times 10^{-24} \mathrm{gm} \mathrm{cm}^{-3}$ we see that as much as $1 / 2 \%$ of the entire interstellar mass is in the form of this organic residue. Since the interstellar hydrogen accounts for about $10 \%$ of the galactic stellar mass, $\mathrm{M}_{\mathrm{Ga}}=10=11 \mathrm{M}$, this leads to the existence in space of $210^{7} \mathrm{M}$ of complex organic material which has been produced by purely abiotic processes.

Grains in molecular clouds

There is abundant evidence that grains in molecular and protostellar clouds have extra accreted mantles of more volatile molecules than the yellow stuff. In general, this extra mantle appears to be dominated by frozen water. From both observational [17] and theoretical [18] considerations one may show that the volatile mantle contains $\sim 60 \% \mathrm{H}_{2} \mathrm{O}$. other molecular constituents, such as $\mathrm{H}_{2} \mathrm{CO}, \mathrm{CO}$, OCS and molecules containing $\mathrm{C} \equiv \mathrm{N}$ have been positively identified from infrared spectra of grains [19, 20, 21]. The classic evidence for grain growth in clouds is based on variations in the wave length dependence of extinction and polarization which point to the presence of larger particles in a correlated way [22].

Contribution of interstellar grains to the prebiotic chemistry of the primitive earth

We see that the space between the stars is filled with an enomous reservoir of complex organic molecules, In fact, by the most optimistic estimates of a galactic planetary mass fraction of $\sim 10^{-3}$ as given by the solar system ratio and, assuming a biomass fraction for each planet of $10^{-10^{2}}$ (as for the earth) we find that the organic matter in space outweighs the galactic biomatter by at least a factor of $10^{10}$.

The question is did or could the interstellar grains play an important role in prebiotic evolution on the earth or elsewhere? Whether they could can be answered in the affirmative. It has been shown that the frequency of passages of the solar system through dense clouds provided a significant source of cosmic matter on the earth before life formed some $3.8 \times 10^{9}$ years ago [23]. However, a much larger and more frequent imput of interstellar dust may be cometary and, by inference, had come in the form of comet impacts - if the theory of comet showers is correct - with an approximate 28 million year period [24]. Comets are suggested to be largely, if not entirely, aggregated interstellar dust [13].

If, say 10 comets per shower in pacted the earth in the period of the early solar system, then the total mass input would have been of the order of the entire bionass of the earth. Using a $20 \%$ complex organic fraction for the comets [25] one can not avoid the fact that early chenical evolution on the earth could not only have been affected by comet impacts it could have been dominated by such matter injected from space. In this context it would appear that the same type of events which later led to extinction of advanced species could have played an important role not only in triggering the first formation of living organisms, it could also have, sporadically, introduced changes on the earth which forced the direction of evolution of surviving species.

\section{Bacterla in space}

The three basic factors in interstellar space which are hostile to microbes are: vacuum, ultraviolet photons, low temperature (of solid particles). The experimental setup which has been designed in the Leiden Astrophysics Laboratory as a simulation of interstellar conditions [26] for the study of chemical evolution of interstellar grains has been used to study the inactivation of bacteria (See Fig. 2). Although inactivation of bacterial spores by ultraviolet radiation has been studied for years under a varlety of conditions these conditions have never sinulated that of the interstellar medium [27]. We chose to consider Bacillus subtilis spores both because of their resistance to vacuun exposure and the existance of a large body of experimental data on inactivation. We have chosen two strains, one of which is relatively radiation resistant, wild type 168 (WT 168, Nester, Marburg), and the other, TKJ 6323 (kindly provided by Prof. Munakata), which is sensitized to irradiation as a result of repair deficiences. Although confirm this relative sensitivity to the $\mathrm{Hg}$ $254 \mathrm{~mm}$ resonance line radiation at room temperature for both vacuum and 1 bar pressure, we find that at $10 \mathrm{~K}$, TKJ 6323 is as strongly resistant to radiation as WT 168 (Figs. 3a, 3b). This trend to less ultraviolet sensitivity of spores at very low temperatures is consistent 


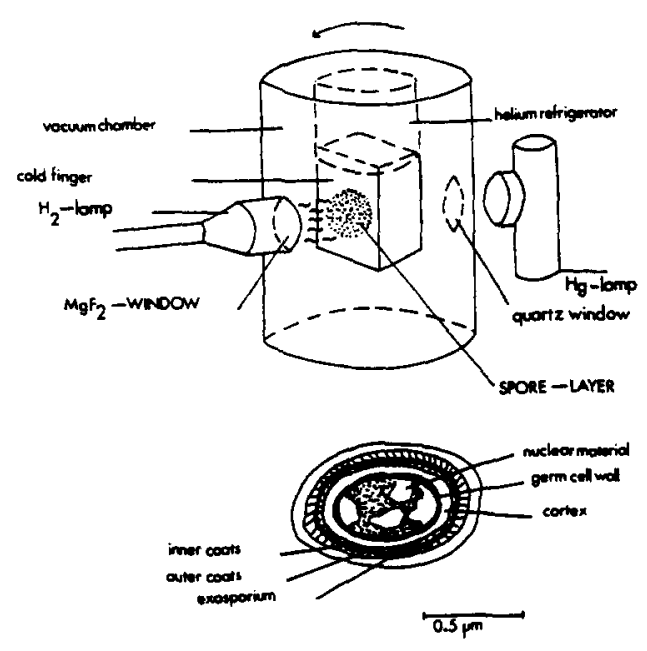

Figure 2: Diagram of apparatus used to irradiate spores. The cold finger on which the spores are deposited is maintained at either $10 \mathrm{~K}$ or room temperature. The quartz window is also used with the $\mathrm{H}_{2}$-lamp.

SPORE CROSS-SECTION

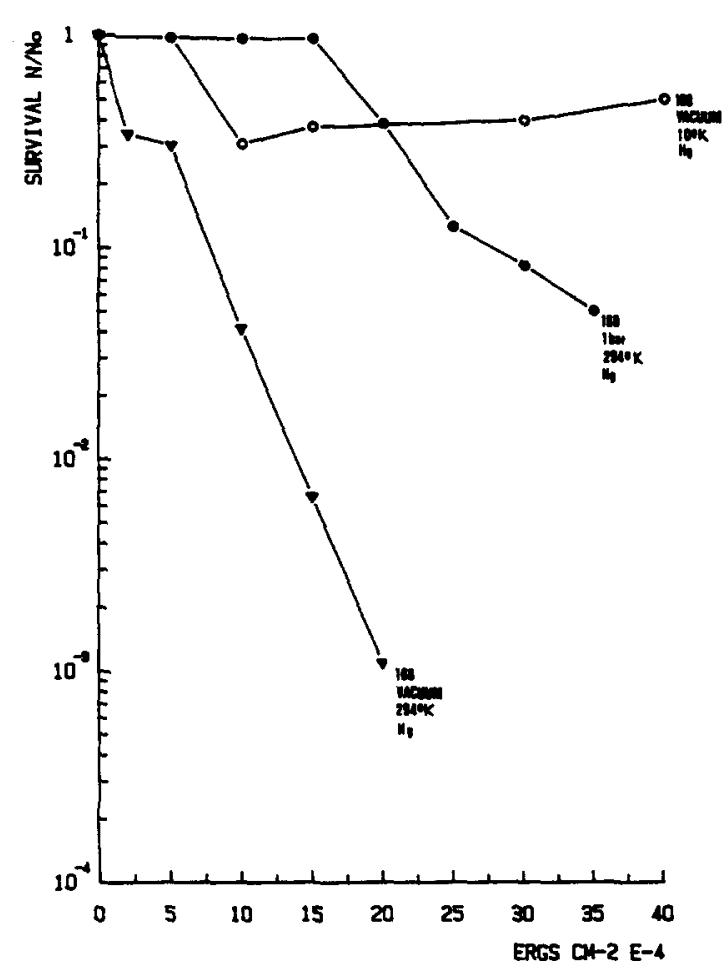

Figure 3a: Inactivation curves for Bacillus subtilis strain WT 168 at $10 \mathrm{~K}$ and at $294 \mathrm{~K}$.

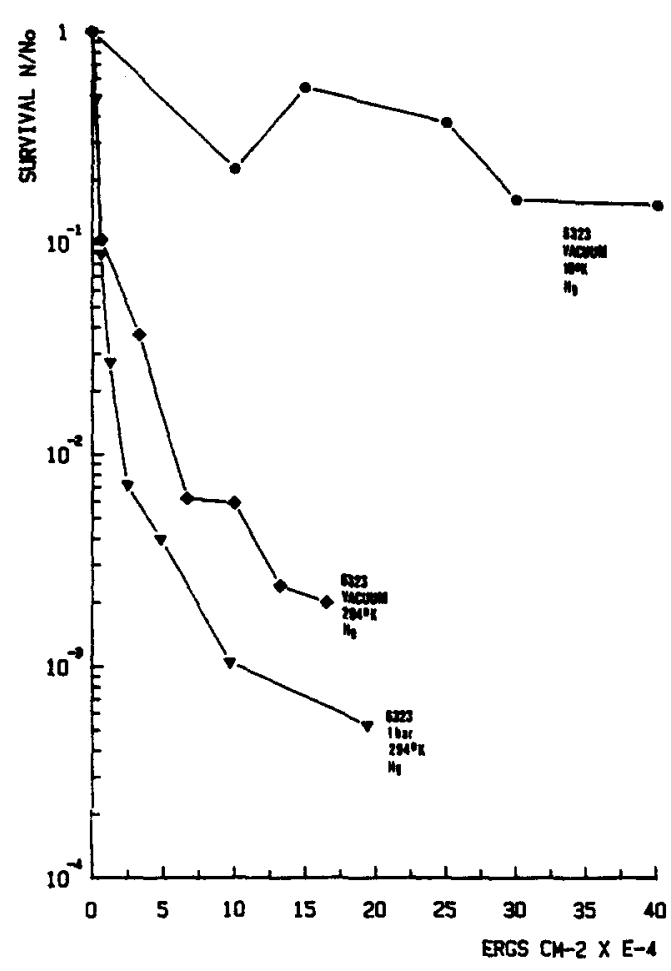

Figure 3b: Inactivation curves for Bacillus subtilis strain TKJ 6323 at $10 \mathrm{~K}$ and at $294 \mathrm{~K}$. 
with the prediction of Ashwood-Smith, et al [28]. As we shall see, if the interstellar radiation consisted only of the Hg resonance line, spores would survive for very long times.

In order to simulate the ultraviolet flux in interstellar space we use the microwave powered $\mathrm{H}_{2}$-discharge lamp which is regularly used in the interstellar dust experiments. At wave lengths shortward of $2000 \AA$ (VUV) the enission of this sourç is peaked at $1600 \AA$ and at $1215 \AA$ (Lyman $\alpha$ ) and if this regton the mean flux is $\sim 1.5 \times 10^{15}$ quanta $\mathrm{cm}^{-2} \mathrm{~s}^{-1}$ as compared with $10^{8}$ quanta $\mathrm{cm}^{-2} \mathrm{~s}^{-1}$ in the diffuse interstellar medium. Between $2000 \AA$ and $3000 \AA$ there is a substantial rise in flux resulting in the fact that the energy output in this region is about 5 times as high as in the vuV. In the interstellar medium this ratio $1 s \sim 1$. Our first suspicion was that the higher energies would be more deleterlous than the lower ones. To our surprise this was not at all the case. In fact (see Fig. 4) we found, by using various filters in the VUV and cutting the VUV off entirely (using a quartz rather than a Mg $F_{2}$ window), that the major damage to our cells was produced by the longer wavelengths (but obviously not $\lambda 254 \mathrm{~nm})$. This result seems to be explainable and will be included in a later report where we exanine relative penetration of ultraviolet photons of different energy in spores and variation in chromophores at different depths.

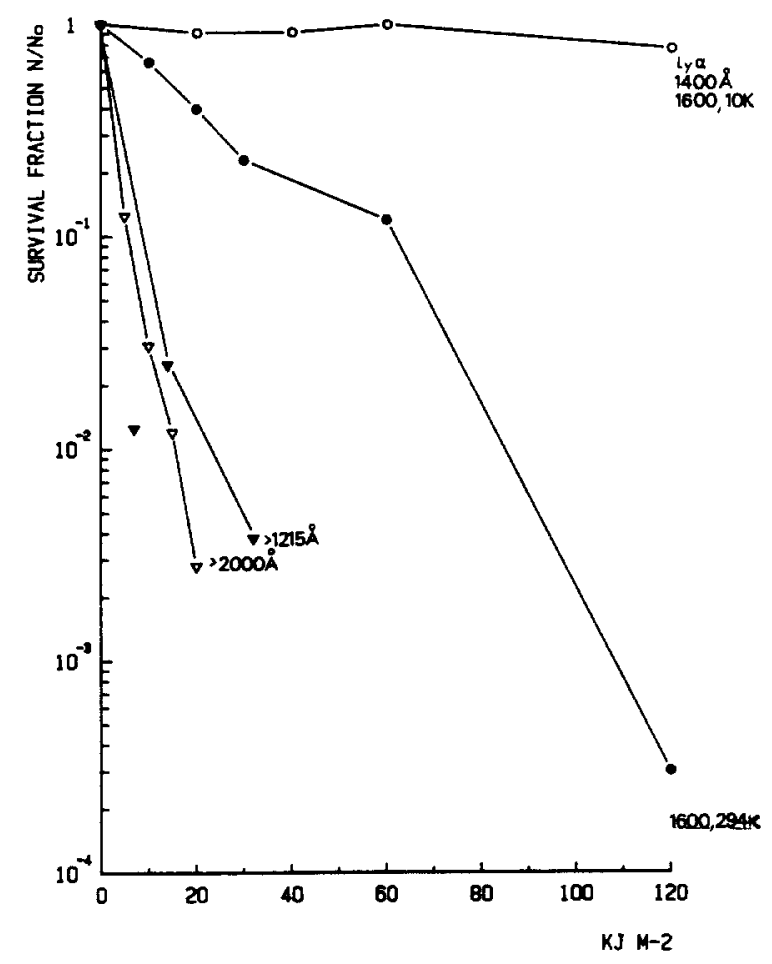

Figure 4: Inactivation curves for Bacillus subtilis as a function of ultraviolet wave length using the $\mathrm{H}_{2}$-lamp. The two lowest curves show the small difference at $10 \mathrm{~K}$ between using the full spectrun $(\lambda>1215 \AA)$ and only the long wavelength portion $(\lambda>2000 \AA)$.

\section{Survival of spores in the ISM}

According to our survival data with the $\mathrm{H}_{2}$ lamp, using a linear regression on the top curve of Fig. 5, it takes a dose of $1 \mathrm{Kj} \mathrm{m}^{-2}$ of vUV to inactivate spores to $\mathrm{F}_{10}$ (10\% survival) at $10 \mathrm{~K}$. Using the full spectrum and noting the fact that the $\mathrm{H}_{2}$ lamp enits 5 times as much energy in the 2000-3000 A range as in the vUV implies that for the diffuse interstellar medium a dose of $F_{10}$ occurs in about 150 years. To derive this result we have used the fact that the flux of vUV by the lamp is such that 1 hour in the laboratory corresponds to about 1000 years in space [26] and the ratio of energy flux in space between $2000 \AA$ and $3000 \AA$ is about equal to that at $\lambda<2000$ \& rather than being 5 times as large. To inactivate spores to $F_{0}$ requires a dose equivalent to 2500 years in the diffuse interstellar medium. At such doses the spores may be presumed to be really dead. 


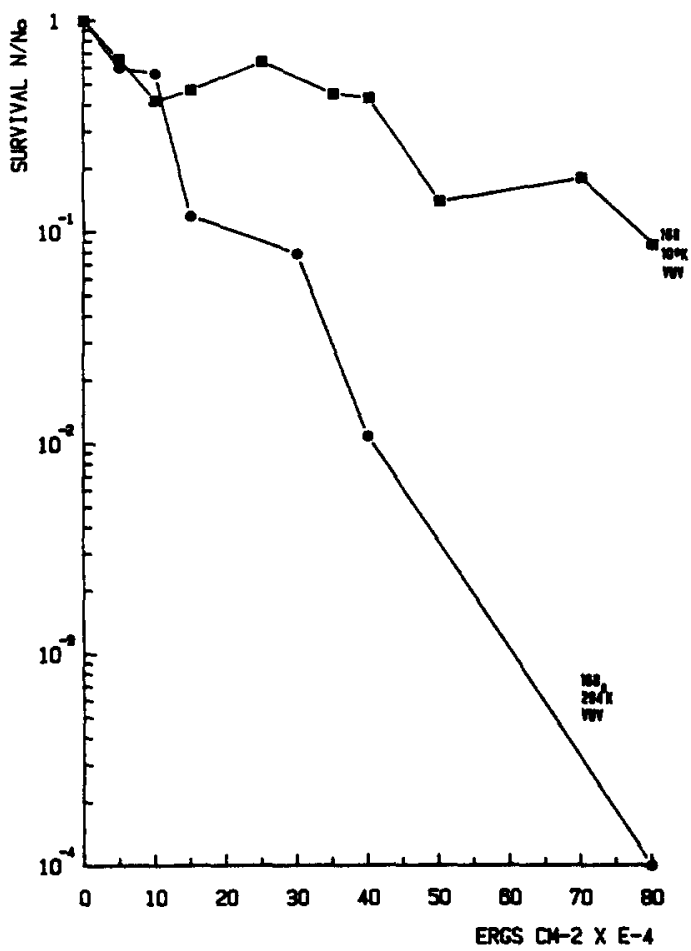

Figure 5: Comparison of inactivation of Bacillus subtilis strain WT 168 by the $\mathrm{H}_{2}-1$ amp full spectrum at $10 \mathrm{~K}$ and $294 \mathrm{~K}$.

Thus the mean survival times for spores in the diffuse region of space is exceedingly short compared with the relevant astronomical time required for transport of a spore from one solar system to another within a molecular cloud. If a spore is caught up in a cloud and carried along with the rest of the material over the distance between neighboring stars of $\sim 0.1$ to 1 parsec ( 0.3 to 3 light year s) [9], this e rresponds to a passage time of $10^{5}$ to $10^{6}$ years. If we asgume that one star in a thousand possesses a solar system we require a survival time of $10^{6}$ to $10^{7}$ years. The next question is: "Can a spore survive as long as a million or ten million years in a molecular cloud?". Two possibilities exist for increased survival time in clouds relative to the diffuse cloud medium:

1) uftraviolet radiation within clouts is generally less by a factor of $10^{3}$ - and more likely $10^{4}$ - than in the diffuse cloud medium.

2) Accretion of atoms and molecules which occurs on spores just as on grains provides a mantle of material which attenutes the ultraviolet radiation. In $\sim 10^{5}$ years a $0.15 \mu \mathrm{m}$ mantle is accreted on a spore in a cloud of density $n_{H}=10^{4} \mathrm{~cm}^{-3}$ leading to an ultraviolet attenuation by at least a factor of 10 for moderate mantle absorptions.

Thus, spores withip a dense cloud have $F_{10}$ values in equivalent interstellar times of the order of $125 \times 10^{3.5} \times 10=10^{6.6}$ years which is just about adequate for viability. of course, should a spore become part of a comet along with the other interstellar matter it could then be protected indefinitely.

Note that we have not yet discussed the question of how surviving spores got into a molecular cloud from a planet. In order for this to occur, the spore must first be injected into the upper atmosphere. Since the effective ultravlolet radiation from the sun is (above the earth's atmosphere) $>10^{10}$ times that of the diffuse I.S.M. [9] the spore must arrive there with a mantle thick enough to provide protection while it is first captured by the cloud and then during the time it takes to be removed from the solar ultraviolet environment to the interstellar ultraviolet enviroment in the cloud. This gives a distance from the sun of $\sim 3000$ a.u. and a passage time of $\sim 10^{10}$ years. An absorbing mantle which attenuates the solar UV by a factor of $\sim 10^{9}$ should be more than adequate not only while the spore is at 
the earth but also during its removal. A mantle of $\sim 0.9$ micron thickness will do this if the imaginary part of its index of refraction is $\mathrm{m}^{\prime \prime}=0.5$.

Finally, with respect to "reentry" of a spore on a non-hostlle planet, we have to postulate again a rather thick protective mantle during its sojourn in the new solar system. One may postulate that this was accreted in the molecular cloud. Another possibility is that the spore is inbedded in cometary debris (interplanetary dust) which penetrates the earth's atmosphere without being overheated iust as is observed for sufficientlv small darticles collected in the upper atmosphere [29].

\section{CONCLUDING REMARKS}

It has been shown that complex organlc residues are produced abiotically in the interstellar medium by ultraviolet photoprocessing of simple ices over time scales of several thousand million years. Spectroscopic evidence in the infrared indicates that a little less than $1 / 10 \%$ of the entire Milky Way is in the form of this organic dust which thus constitutes a mass fraction at least comparable with all planets. Based on the interstellar dust composition of primordial comets, and an estinate of comet impacts, it is shown that the interstellar dust organic molecules could have dominated the chemistry of the early earth. This would imply the possiblitiy that prebiotic evolution was influenced if not directed, by molecules of extraterrestrial origin. Whether there inay be living organisms which survive the hostile interstellar enviroment and can carry life from one planet to another has been shown to depend quantitatively on the degree to which bacterial spores may be shielded from ultraviolet radiation. Laboratory results on inactivation of spores under simulated interstellar conditlons show that without such shielding "life expectancies" of only hundreds of years are posstble which is far less than the millions of years characteristic of interstellar passages. However, attenuation of the ultraviolet within interstellar clouds and within mantles of molecules accreted on the spores may extend inactivation times to tens of million years. Thus, although a part of the question of panspernia appears to be asnwerable on purely sclentific grounds, whether it actually has taken place remains a matter for speculation until one can develop a suitable mechanism for getting viable spores away from a planet and across a solar system in the time a passing cloud takes to carry it out to the interstellar medium. The suggestion that prebiotic evolution was directly influenced, if not dominated, by injection of molecular bullding blocks of extraterrestrial origin is strongly supported if the rate of comet inpacts on the early earth was as large as $1-10$ comets each 28 milition years.

\section{ACKNOWLEDGEMENTS}

This work has been supported in part by NASA grant no. NGR 33-018-148. One of us (P.W.) is grateful to ESA for granting a fellowship to support his research.

\section{REFERENCES}

1. A.P.C. Mann and D.A. Williams, Nature, 283, 721 (1980).

2. W.M. Irvine and $\AA$. Hjalmarson, in Cosmochemistry and the origin of Life, ed. C. Ponnamperuma (Ddrdrecht, Reidel, 113 (1983).

3. A. G.W. Cameron, Sp. Sc1. Rev. 15, 121 (1973).

4. L. Spitzer, Jr. Physical Processes in the Interstellar liedium, J. Wiley and Sons (1978).

5. J.M. Greenberg, In Cosmic Dust, ed. J.A.M. McDonnel1, J. W1ley, N. Y. 187 (1978).

6. J.M. Greenberg and G. Chlewick1, Astrophys. J. 272 (1983).

7. G. Chlewicki, G.P. van der Zwet, L. van IJzendoorn, J.M. Greenberg, and P. Alvarez Astrophys. J., submitted (1984).

8. S.S. Prasad and S.P. Trafadar, Astrophys, J. 267, 603 (1983).

9. C.W. Allen, Astrophyslcal Quantitles, $3^{\text {rd }}$ ed., Athlone Press, London (1973).

10. S. Kwok. J. Roy. Astr. Soc. Can. 74, no. 4, 216 (1980).

11. J.M. Greenbeg, in Submillimetre Wave Astronomy, ed. J.E. Beckman and J.P. Phililps, Cambridge Univ. Press, 261 (1982).

12. L.B. d'Hendecourt, L.J. Allamandola, and J.M. Greenberg, Astron. Astrophys. submitted.

13. J.M. Greenberg, in Conets, ed. L. Wilkening, U. of Arizona Press, 131 (1982).

14. B.T. Draine and E.E. Sal peter, Astrophys. J. 231, 438 (19793/4.

15. E. E. Becklin, K. Matthews, G. Neugebauer and S. P. Willner, Astrophys. J. 220,831 (1978).

16a) J.M. Greenberg, in Infrared Astronomy, ed. G.S. Setti and G. Fazio, D. Reidel, Dordrecht, 173 (1979).

16b) P.F. Roche and D.K. Aitken, Mon. Not. R. Astr. Soc. 208.481 (1984).

16c) J.M. Greenberg and S.S. Hong, in HII Reglons and the Galactic Center, ed. A.F. Moorwood. ESRO SP-105, 221 (1974). 
17) D.C.B. Whittet, M.F. Bode, A.J. Longmore, B.W.T. Balnes and A. Evans, Nature 303,218 (1983).

18) C.E.P.M. van de Bult. J.M. Greenberg and D.C.B. Whitcet. ibn. Not. R. Astr. Soc. accepted (1985).

19) L.H. Lacy, F. Baas, L.J. Allamandola, C. E. P.M. van de Bult, S. E. Persson, P.J. McGregor, C.J. Lonsdale and T. R. Geballe, Astrophys. J. 276, 533 (1984).

20) F. Baas, L.J. Allamandola, T. R. Geballe, S.E. Persson and L.H. Lacy, Astrophys. J. 265, $290(1983)$.

21) T. Geballe, F. Baas, J.M. Greenberg and W. Schutte, Astron. Astrophys., in preparation.

22) D.C.B. Whittet and I. G. van Breda, Astron. Astrophys. 66, 57 (1978).

23) J.M. Greenberg, in Adv. Space Res. Vol. 3 No. 9, 19 (1983).

24) M. Davis, P. Hut, R.A. Muller, Nature 308, 715 (1984) D.P. Whitmire and A.A. Jacks on Nature 308, 713 (1984).

25) J.M. Greenberg, in Asteroids, Comets, Meteors, ed. C.-I. Lagerkvist and H. R1ckman, Uppsala Univ. Press, 259 (1983).

26) W. Hagen, L. J. Allamandol $a$ and J.M. Greenberg, Astrophys. Sp. Sc․․ 65, 215 (1979).

27) G. Horneck, et al. Science, 225, 226 (1984).

28) M.J. Ashwood-Sm1th, J. Copeland and J. Wilcocks on Nature, 217, 337 (1968).

29) D.E. Brownlee in Cosmic Dust, ed. J.A.M. Mc Donnell (J. Wiley and Sons) $295,(1978)$. 